\title{
The Semiclassical and Quantum Regimes of Superradiant Light Scattering from a Bose-Einstein Condensate
}

\author{
G.R.M. Robb $\dagger$, N. Piovella $\ddagger$ and R. Bonifacio $\ddagger$ \\ $\dagger$ Department of Physics, University of Strathclyde, 107 Rottenrow, Glasgow G4 \\ ONG, Scotland. \\ $\ddagger$ Dipartimento di Fisica, Università degli Studi di Milano, INFM and INFN, \\ Via Celoria 16, Milano I-20133, Italy. \\ E-mail: g.r.m.robb@strath.ac.uk, nicola.piovella@mi.infn.it
}

\begin{abstract}
We show that many features of the recent experiments of Schneble et al. [D. Schneble, Y. Torii, M. Boyd, E.W. Streed, D.E. Pritchard and W. Ketterle, Science 300, 475 (2003)], which demonstrate two different regimes of light scattering by a Bose-Einstein condensate, can be described using a one-dimensional mean-field quantum CARL model, where optical amplification occurs simultaneously with the production of a periodic density modulation in the atomic medium. The two regimes of light scattering observed in these experiments, originally described as "Kapiza-Dirac scattering" and "Superradiant Rayleigh scattering", can be interpreted as the semiclassical and quantum limits respectively of CARL lasing.

PACS numbers: $42.50 . \mathrm{Fx}$
\end{abstract}

\section{Introduction}

The study of nonlinear optical phenomena arising from the collective motion of atoms in dynamic optical fields has been an active field of predominantly theoretical research over the last decade. A large fraction of this work has been concerned with the Collective Atomic Recoil Laser (CARL) [1, 2, 3, 4, 5, 6]. As part of the continuing progress in the production and investigation of ultracold atomic gases and Bose-Einstein condensates (BECs), there have been several experiments which have demonstrated the validity of these models and realized some of their predictions. Examples include the observation of collective atomic recoil lasing by a cold thermal gas in a high-finesse cavity [7, 8, and the observation of superradiant Rayleigh scattering by a BEC 9, 10, [11. Superradiant Rayleigh scattering involves the production of pulses of coherently scattered radiation simultaneous with the splitting of the condensate into discrete momentum groups due to atomic recoil. Several theoretical models have been used to describe the evolution of the superradiant scattering process [4, 15, 6, 12, 13, 14, 15], including an extension of the original classical CARL model [1, 2, 3, to include a quantum treatment of the atomic dynamics [5, 6, 13. Recent experimental work by Schneble et al. [16] has shown that in addition to the superradiant Rayleigh scattering process originally observed in [9], there is a second scattering regime termed "Kapiza-Dirac scattering". During superradiant 
Rayleigh scattering, the scattering process involves only emission of scattered photons i.e. absorption of pump photons and emission of scattered (probe) photons. In contrast, during Kapiza-Dirac scattering the scattering process involves both emission and absorption of scattered (probe) photons i.e. absorption of probe photons and emission of pump photons.

In this paper it is shown that many features of the recent experiments of Schneble et al. [16] can be described using a one-dimensional mean-field quantum CARL model, where optical amplification occurs simultaneously with the production of a periodic density modulation in the atomic medium. Using this model, we demonstrate that the two regimes of "Kapiza-Dirac scattering" and "Superradiant Rayleigh scattering" observed in [16], can be interpreted as the semiclassical and quantum limits respectively of CARL lasing. It will be shown that the two regimes are distinguished by the relative size of the gain of the superradiant scattering process and the frequency separation of the absorption and emission peaks. A significant difference between the results presented here and those of other theoretical models of the experiments of Schneble et al. [16, 17] is that in this model the regime of scattering is not determined by the pump pulse duration, so the scattering process does not evolve in time from one to the other.

\section{Model}

The model used to describe the BEC-light interaction is the mean-field quantumCARL model originally derived in [5, 6]. The model is one-dimensional and describes the evolution of a backscattered (probe) field arising from scattering of a pump laser field (assumed to be of constant amplitude) by an elongated BEC. In the experiments of Schneble et. al. [16], the geometry of the experiment is essentially two-dimensional, as illustrated schematically in fig. 2 with emission of two endfire modes from each end of the long axis of the condensate, propagating transversely to the pump laser. If we assume that coupling between the endfire modes (which are much weaker than the pump) is negligible, each endfire mode can be assumed to evolve independently and the atomic motion is one-dimensional.

When the pump laser is sufficiently detuned from the atomic resonance, it leaves the atoms in the internal ground state. Consequently radiation pressure due to absorption and subsequent random incoherent, isotropic emission of a photon, can be neglected. In this detuned regime, coherent scattering of the pump laser is the dominant process. The atoms interact with a laser beam of wave vector $\vec{k}$ and scatter photons of wave vector $\vec{k}_{s}$, recoiling with a momentum $\hbar \vec{q}=\hbar\left(\vec{k}-\vec{k}_{s}\right)$. The atoms, initially scattered randomly into various momentum states, interfere with the atoms in the original momentum state. This creates a matter wave grating having the correct periodicity to further scatter the laser beam in the direction $\vec{k}_{s}$. In an elongated condensate a preferential direction for the scattered photons emerges causing superradiant Rayleigh scattering. Both the matter wave grating and the scattered light are coherently amplified 9 , 16.

In a simplified 1D description of the process along the direction of the atomic recoil momentum $\hbar \vec{q}$, the evolution of the matter wave field $\Psi(\theta, t)$ and of the dimensionless amplitude $a(t)$ of the scattered radiation is determined by the following quantum mean-field CARL model [5, 6]:

$$
i \frac{\partial \Psi}{\partial t}=-\omega_{r} \frac{\partial^{2} \Psi}{\partial \theta^{2}}-i g\left[a e^{i(\theta+\delta t)}-\text { c.c. }\right] \Psi+\beta|\Psi|^{2} \Psi
$$




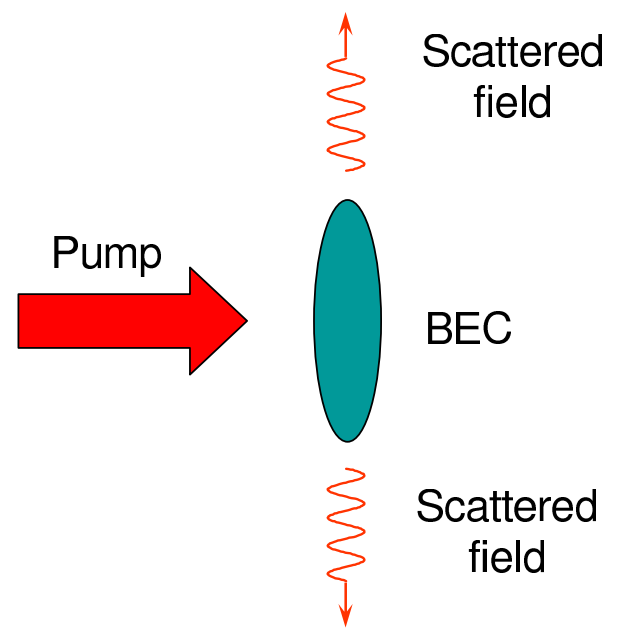

Figure 1. Schematic diagram illustrating the geometry of the superradiant scattering experiments of [16 9

$$
\frac{d a}{d t}=g N \int d \theta|\Psi|^{2} e^{-i(\theta+\delta t)}-\kappa a .
$$

where $\theta=q z$ (with $q=|\vec{q}| \approx \sqrt{2} k$ ), $a=\left(\epsilon_{0} V / 2 \hbar \omega_{s}\right)^{1 / 2} E$ is the dimensionless electric field amplitude of the scattered beam with frequency $\omega_{s}, \omega_{r}=\hbar q^{2} / 2 m$ is the two-photon recoil frequency, $g=(\Omega / 2 \Delta)\left(\omega d^{2} / 2 \hbar \epsilon_{0} V\right)^{1 / 2}$ is the coupling constant, $\Omega=d E_{p} / \hbar$ is the Rabi frequency of the laser field with constant amplitude $E_{p}$ and frequency $\omega=c k, \Delta=\omega-\omega_{0}$ is the detuning from atomic resonance $\omega_{0}, d=\hat{\epsilon} \cdot \vec{d}$ is the electric dipole moment of the atom along the polarization direction $\vec{\epsilon}$ of the laser, $V=A L$ is the volume of the condensate, $A$ is its cross-sectional area, $L$ is its length, $N$ is the total number of atoms, and $\delta=\omega-\omega_{s}$. The matter wave field is normalized such that $\int_{-\infty}^{+\infty} d \theta|\Psi|^{2}=1$. The second term on the right hand side of Eq. (11) is the self-consistent optical lattice, resulting from the interference between the laser and the scattered radiation, whose amplitude is amplified by the matter wave grating described by the first term on the right hand side of Eq. (2). The third term on the right hand side of Eq. (11) describes the mean-field effect of the atom-atom interaction due to binary collisions, where $\beta=8 \pi \hbar q a_{s} N / m A$ and $a_{s}$ is the scattering length. Eq. (2) has been written in the 'mean-field' limit, which models propagation of light with respect to the atoms by replacing the non uniform amplitude by its average value and by adding to the equation a damping term with a decay rate $\kappa \approx c / 2 L$ of the order of the inverse of the photon flight time through the condensate.

Notice that the interaction time $t$ appearing in Eqs. (11) and (2) is the pump field duration, so that our model is suitable to explore both Superradiant Rayleigh scattering ('long pump pulse duration') and self-stimulated Kapitza-Dirac diffraction regime ('short pump pulse duration') investigated in ref. [16]. It will be shown here that the relevant parameter in these experiments is not the pump pulse duration but the characteristic time of the optical/matter-wave amplification process, given by the inverse of the gain rate.

If we assume that the atomic wave function is periodic on the scale of the optical potential, with spatial period $2 \pi / q=\lambda / \sqrt{2}$ which corresponds to a period in $\theta$ of $2 \pi$, 
then we can expand the atomic wave function in a Fourier series

$$
\Psi(\theta, t)=\sum_{n=-\infty}^{\infty} c_{n}(t) e^{i n(\theta+\delta t)}
$$

Furthermore, we assume that the condensate is sufficiently dilute such that $4 \pi \hbar a_{s} n_{s} / m \ll \omega_{r}$, where $n_{s}$ is the average atomic density, so that the atom-atom interaction term in Eq. (11) may be neglected. The effect of the atom-atom term on the collective recoil lasing has been investigated in ref. [18.

Substituting for $\Psi(\theta, t)$ using Eq. (3), it can be shown [5, 6, 12] that Eqs. (11) and (2) can be rewritten as

$$
\begin{aligned}
& \frac{d c_{n}}{d t}=-i n\left(\omega_{r} n+\delta\right) c_{n}-g\left(a c_{n-1}-a^{*} c_{n+1}\right) \\
& \frac{d a}{d t}=g N \sum_{n=-\infty}^{\infty} c_{n} c_{n-1}^{*}-\kappa a
\end{aligned}
$$

In this quantum description, the Fourier expansion (3) is equivalent to expanding the wave function $\Psi(\theta, t)$ in the set of momentum eigenstates with eigenvalues $\vec{p}=(\hbar \vec{q}) n$ and $p_{n}=\left|c_{n}\right|^{2}$ is the probability for an atom to have a momentum $\vec{p}=(\hbar \vec{q}) n$.

In the superradiant regime explored in the experiments of ref. [9, 10, 11, 16, the radiation damping rate $\kappa$ is always much larger than the gain rate and/or the recoil frequency $\omega_{r}$, so that the field amplitude follows the atomic motion adiabatically . Hence, neglecting the time derivative, Eq.(5) yields

$$
a \approx \frac{g N}{\kappa} \sum_{n=-\infty}^{\infty} c_{n} c_{n-1}^{*} .
$$

\section{The semiclassical and quantum limits of the superradiant regime}

In order to obtain the gain coefficient of the superradiant process in the semiclassical and quantum limits from the dynamical equations, let us consider the initial equilibrium state with no field, $a=0$, and all the atoms at rest, i.e. in the momentum state $n=0$, with $c_{0}=1$ and $c_{m}=0$ for all $m \neq 0$. Linearizing around this equilibrium solution and neglecting the small detuning $\delta$ between the laser and scattered frequencies, Eqs. (4) and (6) reduce to the single linear equation for $B=c_{1}+c_{-1}^{*}$ :

$$
\frac{d^{2} B}{d t^{2}}+\omega_{r}\left(\omega_{r}-i G\right) B=0
$$

where $G$ is the superradiant gain

$$
G=\frac{2 g^{2} N}{\kappa}=\frac{\hbar \omega \Gamma \Gamma_{s c} N}{2 A I_{s a t}}
$$

$\Gamma$ is the natural linewidth, $\Gamma_{s c}=\Gamma(\Omega / 2 \Delta)^{2}$ is the Rayleigh scattering rate and $I_{\text {sat }}=c \epsilon \hbar^{2} \Gamma^{2} / 4 d^{2}$ is the saturation intensity. Eq. (8) coincides with the superradiant gain coefficient reported in Eq.(4) of [16].

It is easy to show that Eq.(7) has an unstable solution $B(t) \propto \exp \left[\left(\lambda_{1}+i \lambda_{2}\right) t\right]$, with

$$
\lambda_{1}=\left(\frac{\omega_{r} \sqrt{\omega_{r}^{2}+G^{2}}-\omega_{r}^{2}}{2}\right)^{1 / 2}
$$


and $\lambda_{2}=\omega_{r} G / 2 \lambda_{1}$. Furthermore, it is easy to show, from Eq.(17), that

$$
\frac{\left|c_{1}\right|}{\left|c_{-1}\right|} \approx \frac{G / 2}{\sqrt{\left(\lambda_{1}+G / 2\right)^{2}+\left(\lambda_{2}+\omega_{r}\right)^{2}}}
$$

These expressions show that the instability may have a semiclassical or quantum character, depending on the ratio between the superradiant gain $G$ and the recoil frequency $\omega_{r}$. In fact, in the limit where $G \ll \omega_{r}$, Eqs. (9) and (10) give $\lambda_{1} \approx G / 2$, $\lambda_{2} \approx \omega_{r}$ and $\left|c_{1}\right| /\left|c_{-1}\right| \approx G /\left(4 \omega_{r}\right) \ll 1$. In this limit, the SR process is quantum in nature, with only the lower state $n=-1$ being populated, and the $\mathrm{SR}$ gain is $G$ [5], 12]. In the opposite limit in which $G \gg \omega_{r}$, Eqs. (9) and (10) give $\lambda_{1} \approx \lambda_{2} \approx \sqrt{\omega_{r} G / 2}$ and $\left|c_{1}\right| /\left|c_{-1}\right| \approx 1-\sqrt{2 \omega_{r} / G}$. In this limit, the SR process is semiclassical in nature, with the states $n=1$ and $n=-1$ almost equally populated. In this case, the superradiant gain is given by [4]

$$
G^{\prime}=\sqrt{2 \omega_{r} G}=2 g \sqrt{\frac{\omega_{r} N}{\kappa}} .
$$

The SR gain in the semiclassical limit is always lower than the SR gain in the quantum limit, since $G^{\prime} / G=\sqrt{2 \omega_{r} / G} \ll 1$. Notice also the different dependence of the SR gain on $N$ in the quantum and the classical limits.

\section{Numerical Results}

In order to observe the behavior of the collective scattering process in the nonlinear regime, Eqs. (4) and (5) were integrated numerically with initial conditions $a=0$, $c_{-1}=1 / \sqrt{N}, c_{0}=\sqrt{1-1 / N}$, and $c_{m}=0$ when $m \neq-1,0$ and with parameters corresponding to the semiclassical and quantum regimes of evolution. These parameters correspond to the experiments of $\left[16\right.$ i.e. a ${ }^{87} \mathrm{Rb}$ condensate illuminated by a pump beam of wavelength $\lambda=780 \mathrm{~nm}$ and an intensity of $63 \mathrm{~mW} \mathrm{~cm}^{-2}$. The pump couples to the $5 S_{1 / 2} \rightarrow 5 P_{3 / 2}$ transition which has a natural width $\Gamma=0.37 \times 10^{8}$ $\mathrm{s}^{-1}$, dipole moment $d=2.07 \times 10^{-29} \mathrm{C} \cdot \mathrm{m}$, saturation intensity $I_{\text {sat }}=2.5 \mathrm{~mW} \mathrm{~cm}{ }^{-2}$ and recoil frequency $\omega_{r}=4.7 \times 10^{4} \mathrm{~s}^{-1}$. The condensate had a cigar-shaped form, $15 \mu \mathrm{m}$ in diameter and $200 \mu \mathrm{m}$ in length, so that $\kappa=7.5 \times 10^{11} \mathrm{~s}^{-1}$. Using these parameters, the quantum and classical superradiant gain coefficients of Eqs. (8) and (11) are $G \approx 4.9 \times 10^{6} \times N /|\Delta|^{2}$ and $G^{\prime} \approx 6.8 \times 10^{5} \times \sqrt{N} /|\Delta|$, respectively, where $\Delta$ is the pump-atom detuning in MHz. We assume that $\delta=0$ and that half of the atoms in the condensate participate in each of two superradiant emissions along the main axis of the condensate and that the number of atoms participating in the collective scattering process is $N=10^{5}$ rather than $N=10^{6}$ as quoted for the number of atoms in the condensate in [16]. Qualitative support for this assumption is provided by fig.3A of [16, which shows a large fraction of the condensate atoms do not participate in the coherent superradiant scattering process. From [16, the 'Kapiza-Dirac' experiment was carried out using $\Delta=-420 \mathrm{MHz}$, so that $g=3.2 \times 10^{6} \mathrm{~s}^{-1}$ and the quantum superradiant gain is $G=3 \times 10^{6} \mathrm{~s}^{-1}$. Consequently the collective scattering process is semiclassical in nature, since $G / \omega_{r} \sim 58$. For the experiments in the 'superradiant Rayleigh scattering' regime, $\Delta=-4400 \mathrm{MHz}, g=3.07 \times 10^{5} \mathrm{~s}^{-1}$ and the quantum superradiant gain coefficient is $G=2.5 \times 10^{4} \mathrm{~s}^{-1}$, so that the collective scattering process is quantum-mechanical in nature, since $G / \omega_{r} \sim 0.53$.

Fig. 2 shows snapshots of the atomic momentum distribution at different times for the semiclassical case with $\Delta=-420 \mathrm{MHz}$. It can be seen from fig. 2 (a) that, in 
agreement with our theory, at the beginning of the interaction momentum states $n=1$ and $n=-1$ are the only non-zero momentum states to have significant population, with $p_{1} \sim p_{-1}$, where $p_{n}=\left|c_{n}\right|^{2}$. Eq. (10) predicts a ratio $\left|p_{1}\right| /\left|p_{-1}\right| \approx 0.7$. As time progresses, many momentum states with both positive and negative $n$ are populated. However, the momentum distribution is not symmetric about $n=0$. The average momentum is less than zero, so from momentum conservation there is a net gain of the scattered radiation field, shown in fig 3 due to the difference in photon absorption and emission rates. Fig. 3 shows that amplification of the scattered field in the semiclassical case is simultaneous with the growth of a strong density modulation in the condensate, as represented by the bunching parameter, $b \equiv\left\langle e^{-i \theta}\right\rangle=\sum_{n} c_{n} c_{n-1}^{*}$. The atomic momentum distributions shown in fig. 2] are similar to the time of-flight images observed for the so-called "Kapiza-Dirac scattering" observed in [16], where population of momentum states due to absorption and emission of radiation was observed.
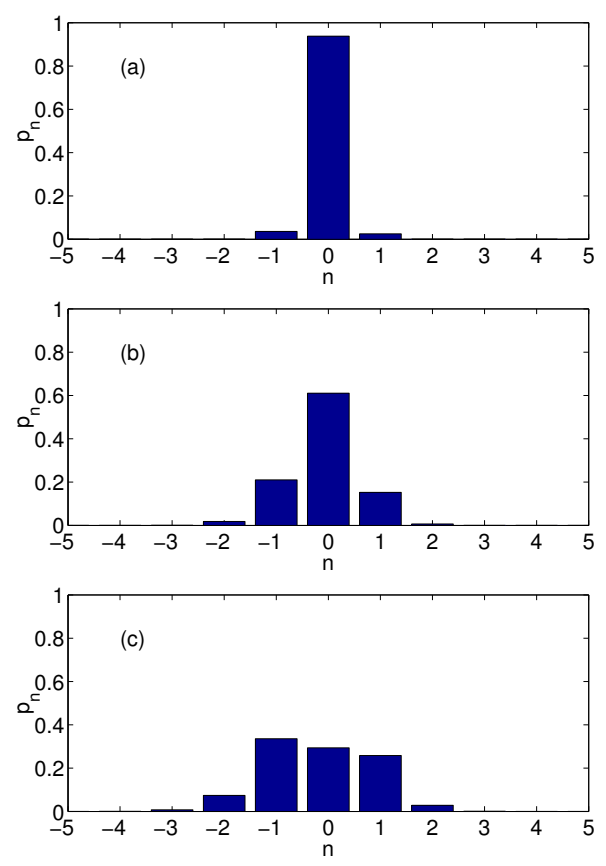

Figure 2. Atomic momentum distribution in the semiclassical regime with $G=58 \omega_{r}$, when (a) $t=11 \mu \mathrm{s}$, (b) $t=15 \mu \mathrm{s}$ and (c) $t=17 \mu \mathrm{s}$.

Fig. 4 shows snapshots of the atomic momentum distribution at different times for the quantum case with $\Delta=-4400 \mathrm{MHz}$. It can be seen from fig. 因(a) that, in agreement with our model, at the beginning of the interaction momentum state $n=-1$ is the only non-zero momentum state to have significant population, and that the population $p_{1} \approx 0$. Eq. (10) predicts a ratio $\left|p_{1}\right| /\left|p_{-1}\right| \approx 0.01$. As time progresses, the atomic population moves sequentially from $n=-1 \rightarrow n=-2 \rightarrow \ldots$ and states with $n>0$ are never populated. This sequential decrease in atomic momentum gives rise to amplification of the scattered radiation field and again occurs simultaneously with the development of a strong density modulation in the condensate, as shown in fig. 囵. In contrast to the semiclassical case, however, this amplification of the scattered 


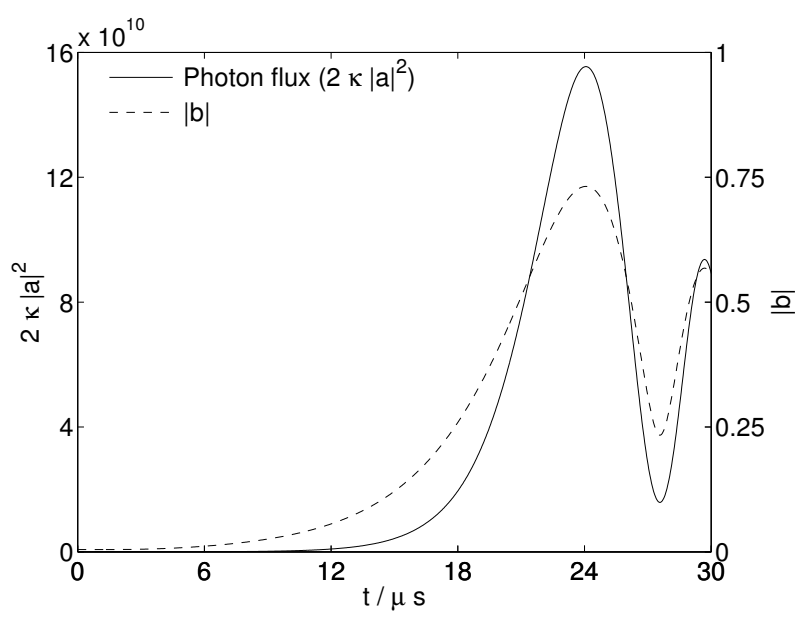

Figure 3. Flux of scattered photons, $2 \kappa|a|^{2}$, and bunching factor, $|b|$, as a function of time in the semiclassical regime with $G=58 \omega_{r}$.

field occurs due to emission of scattered (probe) photons only, and the spread in atomic momenta is much smaller than in the semiclassical case. The atomic momentum distributions shown in fig. [4 are similar to the time of-flight images observed for "Superradiant Rayleigh scattering" in [16, 9, where the atoms attain momentum only in the direction of the pump beam in discrete units of $\hbar \vec{q}$.

\section{Interpretation}

In 16, the Kapiza-Dirac scattering regime and the Superradiant Rayleigh scattering regime are described as the "short-pump pulse" and "long-pump pulse" limits respectively. A problem with this classification is that it implies that for sufficiently long pump pulses the collective scattering makes a transition from Kapiza-Dirac scattering to superradiant Rayleigh scattering, or in the terminology of this paper a transition from the semiclassical to the quantum regime of CARL lasing. The results presented here however do not support this interpretation. They suggest that the distinguishing feature between the two experiments in [16 is not the pump pulse duration, but the atom-field detuning (which differs by an order of magnitude in the experiments of [16]), which determines the atom-field coupling and consequently the timescale of the superradiant scattering process. Rather than the ratio of the pump pulse duration relative to the two-photon recoil time (the inverse of the twophoton recoil frequency), it is the size of the superradiant decay time relative to the two-photon recoil time which determines whether or not the scattering consists of a sequence of emission processes, as observed in the "superradiant Rayleigh scattering" regime [16, 9], or simultaneous emission and absorption processes, as observed in the "Kapiza-Dirac regime".

A simple justification for this argument is as follows: The atoms are assumed to all be in the condensate initially, so that $p_{0}=1$. The frequencies at which the probability of absorption $(n=0 \rightarrow n=1)$ and emission $(n=0 \rightarrow n=-1)$ are maximum are non-degenerate and differ by a frequency of $2 \omega_{r}$. A transition 

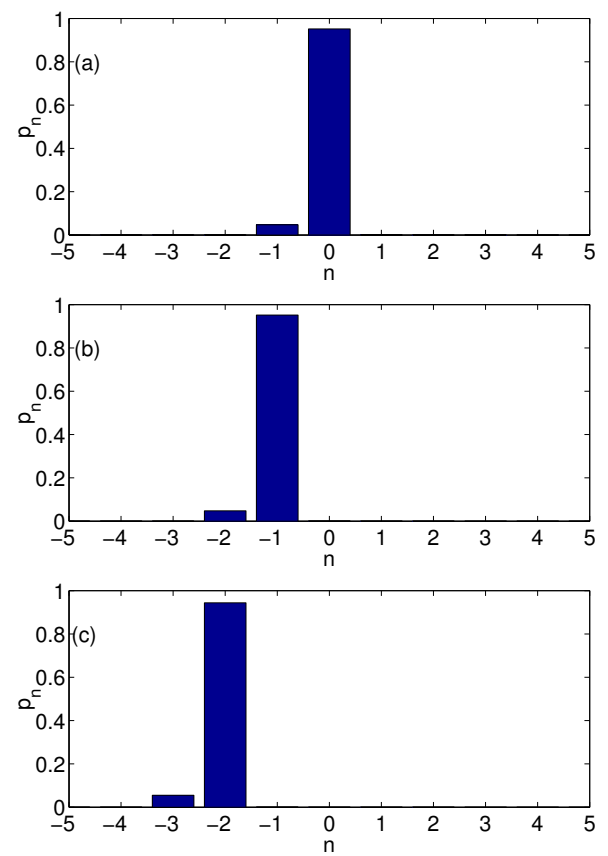

Figure 4. Atomic momentum distribution in the quantum regime with $G=$ $0.53 \omega_{r}$ when (a) $t=0.3 \mathrm{~ms}$, (b) $t=1.0 \mathrm{~ms}$ and (c) $t=1.7 \mathrm{~ms}$.

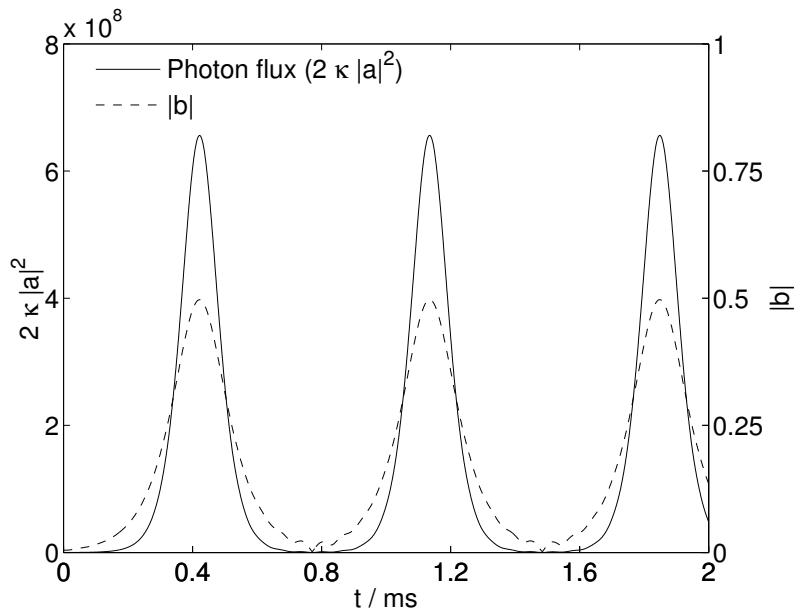

Figure 5. Flux of scattered photons, $2 \kappa|a|^{2}$, and bunching factor, $|b|$, as a function of scaled time in the quantum regime with $G=0.53 \omega_{r}$.

from $(n=0 \rightarrow n=-1)$ will initiate superradiant or superfluorescent decay. The characteristic time of the superradiant decay is $\tau_{s r} \sim G^{-1}$ for $\kappa \gg g \sqrt{N}$ [6], so the spectral width of the superradiant pulse is $\sim G=2 g^{2} N / \kappa$. If the spectral width of the SR pulse is much less than the absorption-emission frequency shift i.e. $g^{2} N / \kappa \leq \omega_{r}$, 
then absorptive transitions will not occur because they are non-resonant and only ( $n=0 \rightarrow n=-1)$ transitions, i.e. absorption of pump photons and emission of probe photons, will occur. Consequently the system evolves in the quantum CARL limit when $G \leq \omega_{r}$. In contrast, if the spectral width of the SR pulse is sufficiently large (i.e. SR decay is sufficiently rapid) that it is much larger than the absorption-emission frequency difference, so that $G \gg \omega_{r}$, then absorptive transitions $(n=0 \rightarrow n=1)$ will be resonant and both emission and re-absorption of probe photons will occur. Consequently the system evolves in the semiclassical CARL limit when $G \gg \omega_{r}$. It should be noted that in contrast to previous explanations [16. 17] of the experimental results in [16], in our argument the duration of the pump pulse is not a significant factor. The reason that the semiclassical CARL or "Kapiza-Dirac" regime can be observed using a short pump pulse in [16] is because the timescale of semiclassical superradiant decay, $\tau_{s r}^{\prime} \sim 1 / G^{\prime}$ (see Eq.(11)) is much shorter than in the "Superradiant Rayleigh Scattering" example as a result of the decreased pump-atom detuning. In Ref. [16] a suppression of quantum SR gain $G$ of Eq. (8) by around two orders of magnitude was observed and attributed in [16] to the short duration of the pump pulse. The CARL model described here explains the reduced gain observed in [16] as a result of the fact that the SR scattering process is evolving semiclassically. Consequently the SR gain is not given by $G$ as given by Eq. (8) but the semiclassical SR gain $G^{\prime}$ as given by Eq. (11). The semiclassical gain, $G^{\prime}$, gives a value consistently smaller than the one predicted by the quantum superradiant gain, $G$. Using the same parameters as those used in figs 4 and 3 , the ratio is $G^{\prime} / G \approx 0.16$.

\section{Conclusion}

It has been shown that many features of the recent experiments of Schneble et al. [16], which show two different regimes of light scattering by the BEC, can be described using a one-dimensional mean-field quantum CARL model. The two regimes of light scattering observed in [16], described as "Kapiza-Dirac scattering" and "Superradiant Rayleigh scattering" in [16, can be interpreted as the semiclassical and quantum limits respectively of CARL lasing. In the semiclassical limit, when $g^{2} N / \kappa \gg \omega_{r}$, the collective scattering process involves both absorption and emission of probe photons, and for sufficiently long times many momentum states are populated simultaneously. In the quantum limit, however, when $g^{2} N / \kappa \leq \omega_{r}$, the collective scattering process involves emission of probe photons only, and a maximum of two momentum states are populated at any time. We provide a simple explanation of these results in terms of a comparison between the frequency separation of probe emission and absorption events and the spectral width of superradiant decay between the initial and recoiling condensates. In contrast to previous models of the experiments in [16], the pump pulse duration is not a significant factor in our interpretation. The results presented here support the view of the BEC-light interaction as a strongly coupled atom-optical system where the atoms and light (and consequently the matter-wave and optical gratings) evolve dynamically and self-consistently. This picture gives a more correct and complete description of the interaction than models in which the dynamics of either the atoms (matter-wave grating) or the optical fields (optical grating) are neglected. 


\section{References}

[1] R. Bonifacio and L. De Salvo, Nucl. Instrum. and Meth. in Phys. Res. A 341, 360 (1994).

[2] R. Bonifacio, L. De Salvo Souza, L.M. Narducci and E.J. D’Angelo, Phys. Rev.A 50, 1716 (1994).

[3] R. Bonifacio, G.R.M. Robb and B.W.J. McNeil, Phys. Rev. A 56, 912 (1997).

[4] N. Piovella, R. Bonifacio, B.W.J. McNeil and G.R.M. Robb, Optics Comm. 187, 165 (2001).

[5] N. Piovella, M. Gatelli and R. Bonifacio, Optics Comm. 194, 167 (2001).

[6] N. Piovella, M. Gatelli, L. Martinucci, R. Bonifacio, B.W.J. $\mathrm{M}^{\mathrm{c}}$ Neil and G.R.M. Robb, Laser Phys. 12, 188 (2002).

[7] G. R. M. Robb, N. Piovella, A. Ferraro, R. Bonifacio, Ph. W. Courteille, and C. Zimmermann, Phys. Rev. A 69, 041403 (2004).

[8] C. von Cube, S. Slama, D. Kruse, C. Zimmermann, Ph. W. Courteille, G. R. M. Robb, N. Piovella, and R. Bonifacio, Phys. Rev. Lett. 93083601 (2004).

[9] S. Inouye, A. P. Chikkatur, D. M. Stamper-Kurn, J.Stenger, D. E. Pritchard and W. Ketterle, Science 285, 571 (1999).

[10] Mikio Kozuma, Yoichi Suzuki, Yoshio Torii, Toshiaki Sugiura, Takahiro Kugam, E.W. Hagley, L. Deng, Science 286, 2309 (1999).

[11] R. Bonifacio, F.S. Cataliotti, M. Cola, L. Fallani, C. Fort, N. Piovella, M. Inguscio, Optics Comm. 233, 155 (2004).

[12] M.G. Moore and P. Meystre, Phys. Rev. A 58, 3248 (1998).

[13] M.G. Moore, O. Zobay and P. Meystre, Phys. Rev. A 60, 1491 (1999).

[14] O.E. Mustecaplioglu and L. You, Phys. Rev. A 62, 063615 (2000).

[15] E.D. Trifonov, Laser Phys. 12, 211 (2002).

[16] D. Schneble, Y. Torii, M. Boyd, E.W. Streed, D.E. Pritchard and W. Ketterle, Science 300, 475 (2003).

[17] H. Pu, W. Zhang and P. Meystre, Phys. Rev. Lett. 91150407 (2003).

[18] N. Piovella, L. Salasnich, R. Bonifacio, nd G.R.M. Robb, Laser Phys. 14, 278 (2004). 